\title{
Is Chi Running a Safer Alternative for those at Increased Cardiovascular Risk?
}

Triya Chakravorty 1 BA (Oxon) \& Elaine Jackson 2 MSc

1 Medical Sciences Division, University of Oxford;

2 Chi Running Practitioner (www.chirunning.uk)

Correspondence to triya.chakravorty@queens.ox.ac.uk

\section{ABSTRACT}

Cardiovascular disease is a global burden, and exercise is a well-recognised route to reducing the risk. Running is an effective form of exercise; however, its intensive nature leads to an increased risk of injury. ChiRunning is an alternative running programme that uses principles of mindfulness adapted from Tai Chi. It focuses on altering running gait, with the goal to decrease injury risk. Due to ChiRunning's recent emergence, data is limited. However, results suggest that ChiRunning improves running gait and may reduce injury risk when compared to traditional methods. Future research with larger sample sizes is required to assess its effectiveness.

\section{Keywords}

ChiRunning, Cardiovascular disease, Alternative running technique

cite as: Chakravorty T, Jackson E. Is ChiRunning a safer alternative for those with increased cardiovascular risk? The Physician 2020 vol 6 (1); v3 Epub 05.05.2020. DOI: 10.38192/1.6.1.4

Introduction

At the beginning of a new year, the minds of many will be on improving health and fitness. It is well known that regular physical activity has several health benefits. Cardiovascular health has been a key focus of biomedical research in the last few decades, and subsequently a substantial body of evidence has emerged linking frequent physical activity with a decrease in cardiovascular mortality and disease (CVD) risk1.

The benefits of exercise are multifaceted, with evidence showing that physically active individuals have improved cardiovascular performance, a more favourable plasma lipoprotein profile, lower blood pressure and higher insulin sensitivity2,3. Blair et al3 showed in a large scale observational cohort study involving over 32000 individuals, that cardiorespiratory fitness levels are inversely correlated to death rates, even in the presence of other predictors of cardiovascular mortality, such as smoking, hypertension and hyperlipidaemia. 
It is clear that the cardiovascular benefits of exercise are vast, and this knowledge is increasingly important when considering that CVD is the leading cause of morbidity and mortality worldwide1. For these reasons, frequent exercise should be an essential part of everyone's daily lives. The question remains as to what form of exercise and 'how much' is most beneficial.

\section{Cardiovascular Benefits of Running}

Running is a well-known form of exercise that is relatively cost effective, requires little to no equipment and can be performed in a wide variety of locations. Due to its popularity, there are several online applications and programmes that runners can use to improve and track their exercise. Importantly, running is a vigorous and intensive form of exercise, which is associated with higher cardiorespiratory fitness levels compared to less intensive activities5,6. Overall, these factors make running a good exercise for beginners and those at a higher CVD risk.

However, the intensive nature of the sport means that there is an increased level of loading on muscles, joints and tendons which leads to increased rate of injury7. Exercise related injury is a serious concern, as it has the potential to severely impact how much exercise a person gets. For example, when in a survey of 2000 individuals, Sallis et als found that the most common reason for discontinuation of an exercise regime was an exercise-related injury. In the last few decades, specialised running programmes have emerged that train individuals to change their running gait in order to reduce risk of injury and therefore increase activity levels. 'ChiRunning' is one such programme 9.

\section{Principles of the ChiRunning Programme}

ChiRunning is a commercially available running training programme that uses principles of mindfulness adapted from the Chinese martial art Tai Chig. This technique focuses on altering running form to introduce a midfoot strike, decrease stride length and increase cadence10. According to the ChiRunning technique, a midfoot strike is characterized as having the runner's heel and ball of the foot touch the ground simultaneously with each foot strike16. Cadence is a measure of the number of strides taken per minute, and a lower cadence, along with decreasing stride length is thought to be related to a greater risk of sustaining injuries 13.

There are six guiding principles of ChiRunning;

- Alignment \& Relaxation,

- Central Movement,

- Cooperating with Force,

- Gradual Progress,

- Body Sensing and

- A Mindful Approach.

ChiRunning differs from traditional running in several ways. For example, the techniques involved aim to decrease the loading forces that are placed on the body during running. By doing this, ChiRunning claims to decrease the risk of injuries sustained during running and therefore promote a greater amount of running activity 10.

A unique aspect of ChiRunning is the integration of mindfulnessi1 into the training programme. Mindfulness and body awareness practices are taught as part of the Chirunning curriculum to both help identify early indicators of injury and increase motivation12. This type of running may be particularly important in a subset of the population who have a higher CVD risk and therefore may benefit more from frequent exercise, such as individuals with prehypertension and hypertension. In conjunction with this, the American Heart 
Association have stated that "alternative" approaches to reducing blood pressure, such as those which increase mindfulness and awareness, may be beneficial adjunctive therapies to prehypertension 13.

When considering how to reduce exercise related injury, ChiRunning provides a novel alternative. If performed correctly, running is a very convenient form of exercise, which may be especially important in individuals who are at an increased risk of CVD. It is of no surprise, therefore, that ChiRunning has been the subject of recent research and investigation.

\section{Impact of ChiRunning}

A number of studies have used motion analysis assessment of running biomechanics to investigate whether running using the ChiRunning technique can alter the running gait to reduce the risk of injury in the way that it claims. Kumar et al 13 conducted a randomised controlled trial pilot study comparing the changes in running biomechanics in individuals with pre-hypertension who received ChiRunning training, to those who received traditional training. Although this study was relatively low-powered, the results showed that ChiRunning training for eight weeks led to improvements in strike index and knee adduction. Therefore, ChiRunning may be a beneficial alternative running technique in regard to reducing the risk of injury. These findings were in accordance with another cross-sectional study that showed that ChiRunners had lower average vertical loading rates and less knee extensor work when compared to runners using the traditional rear foot striking technique14. Importantly, Kumar et al 13 showed with their study that it is possible to train inexperienced individuals the ChiRunning technique in a way that leads to positive results. This has implications for the usefulness of ChiRunning on a larger scale.

In order to investigate the potential impact of ChiRunning on reducing CVD risk in individuals with prehypertension (defined as 120-130/80-90 mmHg) who are at a higher risk of CVD, McDermott et al 15 conducted a similar study, which compared a group receiving eight weeks of ChiRunning training to groups carrying out traditional running methods. Whilst they did not find any significant difference in blood pressure between the groups after the intervention, the ChiRunning group did show a reduction in body mass index (BMI) over time. It is possible that changes in blood pressure may only be discerned over a longer time period and with a larger sample number. However, due to the sensitivity of blood pressure to external factors (such as time of day or week), BMI may be a more stable indicator of efficacy overall. It appears that ChiRunning is a feasible and acceptable training technique for people with prehypertension and therefore should be considered when advising individuals about what lifestyle changes to decrease CVD risk.

\section{ChiRunning - A Coach's Perspective}

ChiRunning is a personal journey and training can vary greatly depending on individual goals. Initially clients attend a 1:1 session to gain insights into the technique, covering aspects such as posture, alignment and the core principles of ChiRunning. Following this some clients choose to continue with regular individual lessons for a bespoke programme or attend small group sessions.

The ChiRunning technique is developed over time, as regular practice enables the runner to progress and feel more comfortable with changes made to their form. At times a runner may need to slow their pace to body sense and prevent over-using muscle groups, the long-term benefits of this are far superior than developing injury and needing time out to recover. Most runners notice their speed increases with ease once they apply the simple technique without forcing the body. The simplicity of ChiRunning allows 
runners to bring mindfulness, enjoyment and a sense of calm to their runs. Over the past twenty years ChiRunning has transformed many runners' technique, enabling them to achieve their goals.

\section{Conclusion}

Cardiovascular disease is a global burden, and exercise is a well-recognised route to reducing the risk. ChiRunning is an alternative approach to running that incorporates ancient ideologies with standard running methods. Through adjustments in running gait, this technique may decrease the risk of exerciserelated injury. Given that such injuries have the potential to seriously impact how much exercise individuals get, ChiRunning may be a valid alternative, especially in individuals who are at an increased risk of CVD.

Due to the relatively recent emergence of this technique, the data surrounding the effectiveness of ChiRunning is limited. However, the results from the handful of studies that do exist are promising, and in the future, larger powered studies with greater sample numbers should be conducted. If the global cardiovascular disease burden continues to follow its current trend, then exploring alternative exercise methods such as ChiRunning may become imperative. it is clear that exercise plans and lifestyle changes can be incredibly hard to stick to. That being considered, ChiRunning may be an alternative exercise method that could motivate people to increase their activity levels in a safe way.

\section{References}

1 Paffenbarger, R. S., Jr., Hyde, R. T., Wing, A. L. \& Hsieh, C. C. Physical activity, all-cause mortality, and longevity of college alumni. N Eng/ J Med 314, 605-613, doi:10.1056/NEJM198603063141003 (1986). 2 Nystoriak, M. A. \& Bhatnagar, A. Cardiovascular Effects and Benefits of Exercise. Front Cardiovasc Med 5, 135, doi:10.3389/fcvm.2018.00135 (2018).

3 Nocon M, Hiemann T, et al. Association of physical activity with all-cause and cardiovascular mortality: a systematic review and meta-analysis. Eur J Cardiovasc Prev Rehabil 2008 Jun; 15(3):239-46

4 Blair, S. N. et al. Influences of cardiorespiratory fitness and other precursors on cardiovascular disease and all-cause mortality in men and women. JAMA 276, 205-210 (1996).

5 Lee, I. M. \& Paffenbarger, R. S., Jr. Associations of light, moderate, and vigorous intensity physical activity with longevity. The Harvard Alumni Health Study. Am J Epidemiol 151, 293-299, doi:10.1093/oxfordjournals.aje.a010205 (2000).

6 O'Donovan, G. et al. Changes in cardiorespiratory fitness and coronary heart disease risk factors following 24 wk of moderate- or high-intensity exercise of equal energy cost. J Appl Physiol (1985) 98, 1619-1625, doi:10.1152/japplphysiol.01310.2004 (2005).

7 van Gent, R. N. et al. Incidence and determinants of lower extremity running injuries in long distance runners: a systematic review. $\mathrm{Br} J$ Sports Med 41, 469-480; discussion 480, doi:10.1136/bjsm.2006.033548 (2007).

8 Sallis, J. F. et al. Lifetime history of relapse from exercise. Addict Behav 15, 573-579, doi:10.1016/0306-4603(90)90059-7 (1990).

9 Huston, P. \& McFarlane, B. Health benefits of tai chi: What is the evidence? Can Fam Physician 62, 881-890 (2016).

10 Dreyer D, D. K. ChiRunning: a Revolutionary Approach to Effortless, Injury-free Running. (2009).

11 Keng, S. L., Smoski, M. J. \& Robins, C. J. Effects of mindfulness on psychological health: a review of empirical studies. Clin Psychol Rev 31, 1041-1056, doi:10.1016/j.cpr.2011.04.006 (2011).

12 Brook, R. D. et al. Beyond medications and diet: alternative approaches to lowering blood pressure: a scientific statement from the american heart association. Hypertension 61, 1360-1383, doi:10.1161/HYP.0b013e318293645f (2013). 
13 Kumar, D. et al. Effects of Form-Focused Training on Running Biomechanics: A Pilot Randomized Trial in Untrained Individuals. PM R 7, 814-822, doi:10.1016/j.pmrj.2015.01.010 (2015).

14 Goss, D. L. \& Gross, M. T. A comparison of negative joint work and vertical ground reaction force loading rates in Chi runners and rearfoot-striking runners. J Orthop Sports Phys Ther 43, 685-692, doi:10.2519/jospt.2013.4542 (2013).

15 McDermott, K. et al. Training in ChiRunning to reduce blood pressure: a randomized controlled pilot study. BMC Complement Altern Med 15, 368, doi:10.1186/s12906-015-0895-x (2015).

16 ChiRunning. 2020. Midfoot Strike, Forefoot Strike or Heel Strike, Which One Is Best?. [online] Available at: <https://www.chirunning.com/blog/midfoot-strike-forefoot-strike-or-heel-strikewhichone-is-best/> [Accessed 5 May 2020] 
